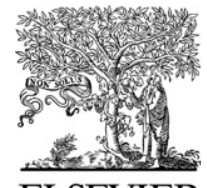

ELSEVIER

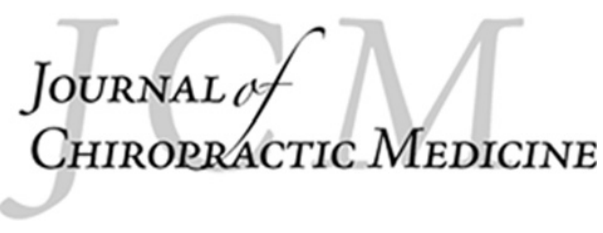

www.journalchiromed.com

\title{
Clinical update
}

\section{Psychometric properties and clinical usefulness of the Oswestry Disability Index}

\section{Michael Vianin DC $^{*}$}

Board member, Swiss Academy for Chiropractic, Department of Postgraduate Studies, Fribourg, Switzerland; staff physician, Clinque Générale, Fribourg, Switzerland; private practice, rue Georges-Jordil 4, 1700 Fribourg, Switzerland

Received 13 April 2008; received in revised form 24 June 2008; accepted 6 July 2008

\author{
Key indexing terms: \\ Disability evaluation; \\ Low back pain; \\ Questionnaires; \\ Reproducibility of \\ results; \\ Chiropractic
}

\begin{abstract}
Objective: Outcome measures with good reliability, validity, responsiveness, and low burden of administration are clinically useful. The Oswestry Disability Index (ODI) is one of the most commonly used outcome measures for individuals with low back pain. Psychometric properties of the ODI will determine the questionnaire's suitability as a useful clinical tool. Methods: A literature search of relevant databases on psychometric evaluation of the ODI was performed. The search was done using the key words disability evaluation, and low back pain, and questionnaires, and reproducibility of results, and the term Oswestry. Inclusion criterion was direct reference regarding psychometric property, interpretability, and burden being included in the abstract.

Results: Eight articles met the inclusion criterion. The ODI shows good construct validity; internal consistency is rated as acceptable; test-retest reliability and responsiveness have been shown to be high; and burden of administration is low.

Conclusions: The ODI is a valid, reliable, and responsive condition-specific assessment tool that is suited for use in clinical practice. It is easy to administer and score, objectifies clients' complaints, and monitors effects of therapy.

(C) 2008 National University of Health Sciences.
\end{abstract}

\section{Introduction}

Clinical decision making relies on valid and reliable outcome measures. Outcome measures determine clients' disability and impairment, choice of therapy,

* Swiss Academy for Chiropractic, Department of Postgraduate Studies, rue Georges-Jordil 4, 1700 Fribourg, Switzerland.

E-mail address: info@mvianin.com. and degree of change over time. To be clinically meaningful, an outcome measure must be easy to complete and score, valid, reliable, and responsive. ${ }^{1}$

The Oswestry Disability Index (ODI) is one of the most commonly used outcome measures for individuals with low back pain (LBP) $)^{2-4}$ The ODI is a selfadministered questionnaire that requires 5 minutes to complete and 1 minute to score. Scores are associated with degree of disability ranging from minimal to 
bedbound. The ease of administering, scoring, and interpreting renders the ODI a potentially meaningful tool in clinical practice.

Psychometric characteristics (validity, reliability, and responsiveness) of the ODI are the final determinants of the questionnaire's suitability as a clinically useful measure. Validity expresses the ability of a tool to measure what it intends to measure, whereas reliability describes a tool's ability to be consistent, to have small errors of measurement, and to differentiate among clients. $^{5} \mathrm{~A}$ valid and reliable outcome measure is clinically relevant only if it possesses the ability to assess change over time (sensitivity to change). ${ }^{5}$ Even more clinically meaningful is the measurement of change over time that is important to clients (responsiveness). ${ }^{5}$

The determination of the appropriateness of the ODI as an outcome measure used to direct clinical decision making requires a critical review of its psychometric properties, interpretability, and burden of administration.

\section{Methods}

A search of the literature was performed using the key words disability evaluation, and low back pain, and questionnaires, and reproducibility of results, and the term Oswestry. The search was performed on Medline, CINAHL, EMBASE, and MANTIS. The search engines were selected for their relevance to the musculoskeletal field. The search yielded 27 citations of which 12 were not duplicates. Citations were kept if direct reference regarding psychometric property, interpretability, and burden was included in the abstract. Eight articles met the inclusion criterion and were included for review and analysis. ${ }^{1-4,6-9}$

\section{Results}

The ODI's psychometric properties have been well established. The ODI correlates with other outcome measures aiming at measuring disability due to LBP. ${ }^{1-4,7}$ Validation of the Pain Disability Index, the Low Back Outcome Score, the Manniche Scale, the Aberdeen Score, the Curtin Scale, and a functional capacity evaluation was performed with the ODI. ${ }^{2,3}$ Therefore, the ODI shows good construct validity because it is consistent with some and was used as the standard of comparison for other outcome measures assessing LBP-induced disability.
Internal consistency has been shown to be of acceptable level by different authors. Cronbach $\alpha$ ranges from .71 to $.87 .2,3$

Test-retest reliability has been shown to be high. Values range from $r=0.83$ to 0.99 and vary according to the time interval between measurements. ${ }^{1-3}$ The longer the wait between repeated measures is, the lower the score becomes. Intraclass correlation coefficient values from 0.84 to 0.94 have been reported. ${ }^{1,7}$

Responsiveness has been reported to be high. The most common method for measuring responsiveness found in the current literature search was a receiver operating characteristic curve. The area under the curve can be used as a quantitative method to assess true change and can be interpreted as the probability of identifying clients who have undergone true improvement. The receiver operating characteristic can also be used to estimate the minimum clinically important difference (MCID). Values for the area under the curve range from 0.723 to $0.94 .3,4,6,7$ The MCID has been reported to be between 4 and 10.5 points. ${ }^{1,2,6,7}$ The consensus calls for a minimal change of 10 points to be clinically significant. ${ }^{1,6}$

\section{Discussion}

The ODI is a valid, reliable, and responsive condition-specific assessment tool that has withstood the test of time and scrutiny. ${ }^{3}$ So far, it has been used mostly in chronic and severely disabled populations, but shows good indicators for the assessments of less severe complaints. ${ }^{2}$

Administration burden is small. The questionnaire takes 5 minutes to complete and less than 1 minute to score. ${ }^{3,6}$ The suggested time interval for readministration of the ODI is 6 weeks. ${ }^{6}$ The scoring system is well described and easy to adopt. ${ }^{2,4,6}$ The ODI has been modified over the course of its existence so that the burden of selection is placed upon clinicians. The authors of the ODI have addressed this dilemma by offering an updated version (Version 2.0) of the questionnaire. $^{2}$ Version 2.0 is recommended for general use. ${ }^{2,3}$ The ODI includes a section on sexual life that has placed the burden on selective clients. Omitting this section does not alter the psychometric characteristics of the questionnaire, and scoring can easily be adjusted for the absence of that information. ${ }^{2}$

Interpretability of the ODI is good. The scoring system includes a description of degrees of disability relating to scores on the ODI. Scores from $0 \%$ to $20 \%$ 
indicate minimal disability; $20 \%$ to $40 \%$, moderate disability; $40 \%$ to $60 \%$, severe disability; $60 \%$ to $80 \%$, crippled; and $80 \%$ to $100 \%$, bedbound or exaggerating. ${ }^{6}$ Changes in scores can infer meaning because of the well-defined responsiveness and MCID. Therefore, the ODI can be used to assess response to treatment. ${ }^{9} \mathrm{~A}$ limitation to the ODI's interpretability might be the lack of superior responsiveness compared with general health questionnaires. Condition-specific outcome measures are not more responsive than their general health counterparts, ${ }^{1,4}$ but lack other facets of disability (emotional, social, and psychologic factors). Although use of condition-specific measures is acceptable in the general population, supplementation with general health measures such as the Short Form 36 is advisable, especially in multidisciplinary management of LBP. ${ }^{4}$

Comparison with other condition-specific outcome measures such as the Roland Morris Questionnaire and the Quebec Back Pain Disability Scale (Quebec), as well as comparison with general health questionnaire such as the Short Form 36, yields comparable results in terms of validity, reliability, and responsiveness. ${ }^{1,3,7-9}$ Test-retest reliability is slightly higher for the ODI compared with the Roland Morris Questionnaire and Quebec. ${ }^{1,7}$ Responsiveness is higher for the ODI than for the Quebec. ${ }^{7}$ The ODI seems to have a slight advantage in the assessment of chronic and more severely disabled clients ${ }^{2,3}$ and is more sensitive for patients showing improvement compared with unchanged clients. ${ }^{9}$

\section{Conclusion}

The ODI is an acceptable tool to measure disability caused by LBP in the general population. The measurement of disability is an important part of
LBP's assessment and management. The choice of the most appropriate outcome measure should rely on the nature of the condition, the type of data desired by the clinician, and environmental factors. The ODI is well suited for use in busy clinical settings that focus on the assessment of impacts caused by chronic LBP or gains after interventions. It is easy to administer and score, objectifies clients' complaints, and monitors effects of therapy.

\section{References}

1. Davidson M, Keating JL. A comparison of five low back disability questionnaires: reliability and responsiveness. Phys Ther 2002;82(1):8-24.

2. Fairbank JC, Pynsent PB. The Oswestry Disability Index. Spine 2000;25(22):2940-52 [discussion 52].

3. Roland M, Fairbank J. The Roland-Morris Disability Questionnaire and the Oswestry Disability Questionnaire. Spine 2000;25(24):3115-24.

4. Walsh TL, Hanscom B, Lurie JD, Weinstein JN. Is a conditionspecific instrument for patients with low back pain/leg symptoms really necessary? The responsiveness of the Oswestry Disability Index, MODEMS, and the SF-36. Spine 2003;28(6): 607-15.

5. Finch E, Brooks D, Stratford P, Mayo N. Physical rehabilitation outcome measures: a guide to enhanced clinical decision making. 2nd ed. 2002.

6. Davidson M, Keating J. Oswestry Disability Questionnaire (ODQ). Aust J Physiother 2005;51(4):270.

7. Fritz JM, Irrgang JJ. A comparison of a modified Oswestry Low Back Pain Disability Questionnaire and the Quebec Back Pain Disability Scale. Phys Ther 2001;81(2):776-88.

8. Reneman MF, Jorritsma W, Schellekens JM, Goeken LN. Concurrent validity of questionnaire and performance-based disability measurements in patients with chronic nonspecific low back pain. J Occup Rehabil 2002;12(3):119-29.

9. Taylor SJ, Taylor AE, Foy MA, Fogg AJ. Responsiveness of common outcome measures for patients with low back pain. Spine 1999;24(17):1805-12. 